\title{
Unidades estructurales y su importancia en la ordenación del territorio ecuatoriano
}

\author{
Structural units and their importance in the \\ management of the Ecuadorian territory
}

Por:

Fernando Pauta Calle

Jaap van der Zee

Universidad de Cuenca

Recibido:

15 de febrero 2012

Aceptado:

15 de Marzo 2012

\section{Resumen:}

El objetivo de este estudio es mostrar la utilidad conceptual y metodológica que tiene la definición de las "unidades estructurales" en la determinación de la capacidad de acogida del medio físico para las actividades humanas, en el marco de la redacción de los planes de ordenación territorial. Para tal efecto, en la introducción se sitúa como punto de partida una de las metodologías propuestas por el profesor Domingo Gómez Orea y las restricciones que presenta para tal propósito desde un enfoque más sistemático.

En calidad de contexto se define a la ordenación territorial, se enuncian los pasos del proceso metodológico a seguir para la elaboración de un plan de ordenación territorial y en particular para el establecimiento de la capacidad de acogida del medio físico y como parte de este componente, de las ya mencionadas unidades estructurales, pero también de las "unidades ambientales", destacando las bondades que tiene en principio encontrar estas últimas, siguiendo el método de Gómez Orea. Luego se conceptualiza a las unidades estructurales y se explica cómo pueden ser delimitadas a distintos niveles de detalle. Seguidamente se expone la función físico-ambiental de las unidades estructurales y su diferencia con las ambientales y asimismo su función socioeconómica. Finalmente se ilustra su definición con una aplicación concreta. El resultado principal señala que el discernimiento de la capacidad de acogida del medio físico para las actividades humanas se vuelve más idóneo, riguroso y consecuente con sus características si se sustenta en las unidades estructurales, sin perjuicio de la posterior definición, con intenciones de análisis y diagnóstico de las unidades ambientales sugeridas por Gómez Orea en atención a las actividades que soporta en un momento dado dicho medio.

Palabras clave: Metodología, unidades estructurales, ordenación del territorio, Ecuador.

\section{Abstract:}

The objective of this study is to show the conceptual and methodological utility that has the definition of "structural units" in the determination of the reception capacity of the physical environment for human activities, in the framework of the drafting of the territorial organization plans. For this purpose, in the introduction is located as a starting point one of the methodologies proposed by Professor Domingo Gómez Orea and the restrictions presented for that purpose from a more systematic approach.

Town and country planning is defined as a context, the methodological process are set out for the elaboration of a land management plan and in particular for the establishment of the capacity of the physical environment and as part of this component, the aforementioned structural units, but also the "environmental units", highlighting the advantages that has in principle to find the latter, following the method of Gómez Orea.

Then the structural units are conceptualized and explained how can be defined at different levels of detail. Is then exposed the environmental function of structural units and their difference with the environmental and also its socio-economic function. Finally shown its definition with a specific application. The main result points to the discernment of the reception capacity of the physical environment for human activities becomes more appropriate, rigorous and consequent with its features if it relies on structural units, without prejudice to the subsequent definition, with intentions of analysis and diagnosis of the environmental unit suggested by Gómez Orea in attention to activities that support eventually that medium.

Keywords: Methodology, structural units, territorial organization, Ecuador. 


\section{Introducción:}

Este trabajo pretende exponer resumidamente las reflexiones conceptuales y metodológicas generadas al interior de la asignatura Curso-Taller de la Maestría de Ordenación Territorial que lleva adelante la Facultad de Arquitectura y Urbanismo de la Universidad de Cuenca a través de su Centro de Postgrados -Julio/Diciembre 2011-, sobre un aspecto trascendental de la formulación de los planes de ordenación territorial, como lo constituye la definición de unidades del medio físico -esto es, de unidades relacionadas con los elementos y procesos del ambiente natural, tal y como se hallan en un momento dado-, en tanto condición necesaria para su valoración y la posterior determinación de la capacidad de acogida de las mismas, para las diferentes actividades de la población consideradas en el plan.

Domingo Gómez Orea, profesor de esta Maestría, impartió entre las metodologías desarrolladas para el efecto en su obra Ordenación Territorial" (1), aquella que en función de los resultados del inventario del medio físico lleva a la integración de la información sectorial obtenida y a la definición de las unidades ambientales; no obstante, la superposición de todos los factores inventariados conduce a un excesivo y engorroso fraccionamiento del territorio, por ello suele resultar útil simplificarlo recurriendo a la superposición de aquellos factores con mayor carga explicativa: la geomorfología (que describe materiales, formas y procesos del medio inerte y sintetiza sus relaciones), la vegetación natural y cultivos (que explica las condiciones ambientales determinantes de la vida y sintetiza las relaciones entre el medio biótico y abiótico) y los usos del suelo (que explican el devenir histórico de las formas de utilización y aprovechamiento del suelo y sus recursos)" (2).

Sin dejar de apreciar este camino, es pertinente señalar que éste no diferencia con respecto al ambiente natural sus atributos permanentes o mejor casi permanentes -como el suelo, la vegetación y la geomorfología- y que definen en última instancia su capacidad de acogida para las actividades humanas, de los variables en el tiempo, como lo son justamente estas últimas, expresadas por el factor ambiental uso del suelo; asunto que, como se observará, tiene ya una gran incidencia en el esquema metodológico mismo. Pero, por otra parte, el enfoque en consideración no permite establecer en toda su extensión las interacciones e interrelaciones, con origen en esos atributos físico-ambientales casi permanentes, entre unidades ambientales identificadas al interior de una misma circunscripción, de un cantón por ejemplo; así como las existentes con las unidades de las circunscripciones contiguas, pues ellas, las aludidas interacciones e interrelaciones, van más allá de los límites político-administrativos y de allí la necesidad de incorporar al referido esquema la definición previa de las unidades estructurales para superar estas limitaciones. Exponer esta opción metodológica es el propósito central de este trabajo.

\section{Contexto}

Entre las muchas interpretaciones de las que ha sido motivo la ordenación territorial, hacia el futuro y desde la perspectiva de su dimensión planificadora, ella también significa intervenir en el universo como lo percibimos, a fin de crear mayores niveles de bienestar y seguridad para la población, como en el caso del Ecuador, reflejados en los doce objetivos nacionales que constan en el Plan Nacional para el Buen Vivir. A su vez, en el ámbito de las competencias vinculadas a esta materia, las normas de la Constitución consignadas en los Artículos 262, 263, 264 y 267 otorgan a los gobiernos autónomos descentralizados regionales, provinciales, municipales y parroquiales rurales, respectivamente, la ordenación territorial de sus circunscripciones; en tanto que al Estado central y en relación a la incidencia territorial de sus competencias exclusivas, de conformidad con el artículo 10 del Código Orgánico de Planificación y Finanzas Públicas, le corresponde desarrollar una "Estrategia Territorial Nacional". Vale destacar que en el caso de los gobiernos municipales su competencia se refiere también al aspecto básico de la ordenación territorial, como es la regulación del uso y la ocupación del suelo cantonal.

El territorio o universo que acoge las actividades humanas puede ser imaginado de diferentes maneras, por ejemplo: un modelo que comprende el ámbito social (la sociedad), la naturaleza o medio físico (ambiente natural) y el producto de la interacción entre éstos dos o sea el ámbito económico (ver Gráfico $N^{\circ} 1$ ), como también las interacciones entre todos sus componentes y elementos. Dicha figura además muestra un ejemplo -en este caso del sector industrial- de la intervención humana, que pretende explicitar cómo las intervenciones en cada sector afectan a todos los componentes y elementos del modelo. Esta complejidad de procesos es conocido popularmente como el "medio ambiente". 


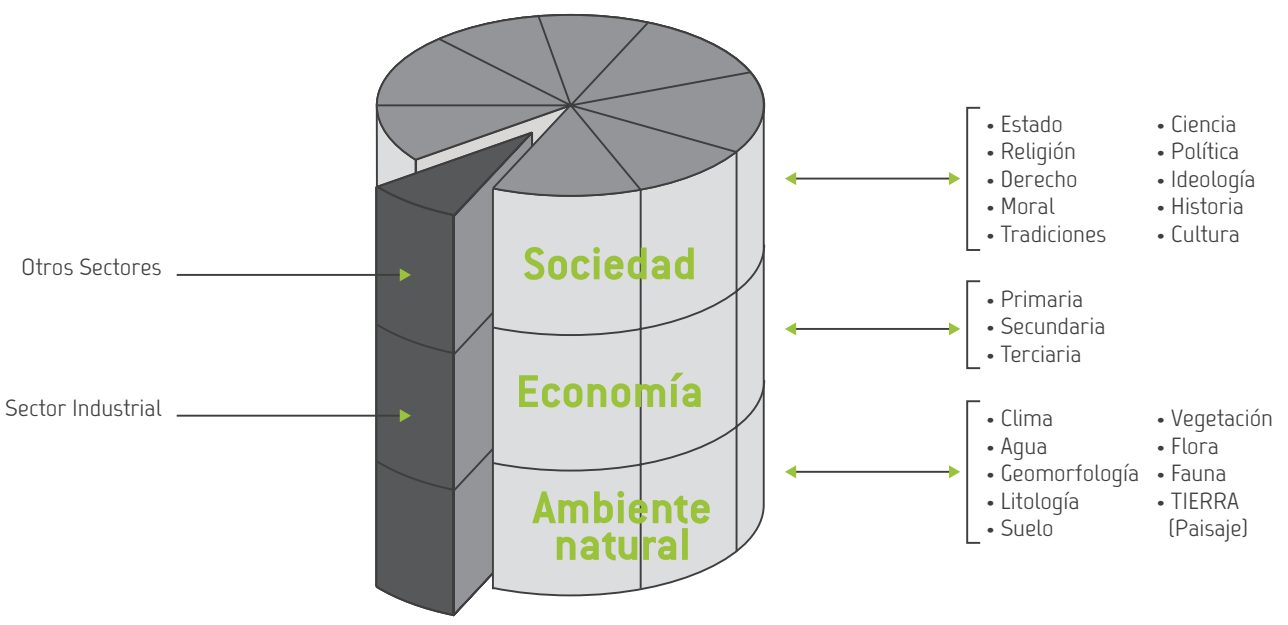

Gráfico $N^{\circ}$ 1: Ejemplo conceptual de medio ambiente Fuente y elaboración: Propia

La virtud de la metodología desarrollada por Domingo Gómez Orea para la formulación de la ordenación del territorio, ya comentada en líneas anteriores, es que sabe captar este universo a través de un enfoque de sistemas, lo que permite tanto una sobrevista más clara del mundo que habitamos, como el uso de métodos de análisis que de forma integral conducen a encontrar sus problemas, causas y efectos, interrelaciones y potencialidades. De la misma manera, la metodología de Gómez Orea otorga también la sobrevista del territorio a diferentes niveles de detalle, de lo macro a lo micro. En cuanto al nivel macro, este se puede alcanzar en un tiempo relativamente corto y con un mínimo de información y medios de trabajo. El enfoque idóneo para la construcción de una propuesta de ordenación territorial, esto es, que comprenda al conjunto de todas las variables, debe imaginar una fórmula de enclaustre, empezando a nivel internacional -factores externos que influyen en el futuro del país-, seguido por el nivel nacional -actores que influyen a lo interno del país-, nivel regional, provincial, cantonal, parroquial y las comunidades.

El objetivo final de la ordenación territorial es la ejecución de un Plan de Ordenación Territorial (POT), y su redacción demanda el cumplimiento de los siguientes pasos: 1) construcción del modelo territorial actual (MTA) y sus tendencias; 2 ) identificación participativa de opciones para el desarrollo, tomando en cuenta las limitaciones presupuestales y las políticas vigentes; 3) priorización de opciones escogidas; 4) construcción del modelo de futuro (MTF) y 5) elaboración de las determinaciones normativas y de inversión del plan, incluyendo en estas últimas los proyectos de corto plazo a nivel de detalle.

Así, la construcción del MTA y sus tendencias requiere de un análisis del territorio o del sistema. En este sistema se reconocen a su vez seis subsistemas: 1 ) el ambiente natural; 2 ) el social y cultural; 3) el económico; 4) el de asentamientos humanos -incluso los equipamientos y servicios-; 5) el de movilidad y conectividad y 6) el de gestión territorial -marco legal e institucional-.

De los seis subsistemas, generalmente se analiza en primera ins- tancia el ambiente natural, ya que éste que sirve de base para la definición del territorio y por cuanto entonces es el menos susceptible de negociación, en la medida que se refiere a elementos y procesos vinculados a la vida, como el aire y el agua por ejemplo. Pero considerando que además necesitamos alimentos, energía, materiales de construcción, minerales, asentamientos humanos y redes de comunicación, es fundamental escoger los lugares que ofrecen las mejores condiciones para cada actividad humana en términos de sostenibilidad y sustentabilidad, lo que remite a establecer lo que se ha dado en Ilamar "la capacidad de acogida del ambiente natural".

La capacidad de acogida, desde un enfoque alternativo que considere expresamente los atributos físico-ambientales que lo especifican y las interacciones e interrelaciones que se generan entre unidades ambientales, podría hallarse mediante la siguiente secuencia técnica: 1) delimitación de los unidades estructurales; 2) delimitación de las unidades ambientales; 3) valoración de las unidades ambientales y 4) determinación propiamente dicha de la capacidad de acogida. Las unidades estructurales representan "el cimiento de la casa", vale decir, el fundamento del MTA y definen su potencial, sus limitaciones y normas de manejo y posteriormente, la construcción del MTF. Las unidades estructurales se pueden delimitar a diferentes niveles de detalle, es decir: macro ( región), procesos de base (subregión, incluso cuencas y subcuencas), reconocimiento (cantones), semi-detalle (parroquias) y detalle (comunidades). Justamente, el nivel de detalle dependerá de los objetivos específicos de la ordenación territorial. 


\begin{tabular}{|c|c|c|c|c|c|}
\hline Nivel detalle & Territorio & Escala & Suelos & Vegetación & Geomorfología \\
\hline $\begin{array}{c}\text { Prelevantamiento } \\
\text { (Prediagnóstico) }\end{array}$ & Región & $1: 250.000$ & Orden & Macrogrupo & Sistema montañoso \\
$\begin{array}{c}\text { Procesos de base } \\
\text { (Diagnóstico) }\end{array}$ & Provincia & $1: 100.000$ & Suborden & Ecosector Ecosistemas & $\begin{array}{c}\text { pie de monte, valle } \\
\text { Reconocimiento }\end{array}$ \\
Cantón & $1: 50.000$ & Gran Grupo & Asociaciones de bosques & $\begin{array}{c}\text { meseta, colina, } \\
\text { terraza }\end{array}$ \\
Prefactibilidad & Parroquia & $1: 10.000$ & Familia & Biotopos & $\begin{array}{c}\text { cárcava, meandro } \\
\text { ondulado, talud }\end{array}$ \\
\hline
\end{tabular}

Cuadro $\mathbf{N}^{\circ}$ 1: Elaboración de unidades estructurales a niveles compatibles con el detalle de la

\section{¿Qué son las unidades estructurales y cómo se elaboran?}

Una "unidad estructural", llamada también "unidad territorial" o "unidad de tierra", según el concepto de tierra de Zonneveld, I.S. (3). La unidad de tierra se caracteriza por la dinámica -es decir las interacciones y las interrelaciones-, de los ocho atributos físico-ambientales: clima, agua, litología, geomorfología, suelo, vegetación, flora y fauna, en el mismo espacio delimitado; además se define según el uso dado por el hombre (ver Figura $N^{\circ} 2$ ). Así, la unidad estructural o unidad de tierra, podría ser denominada como unidad ecológica, geomorfológica, de suelo, etcétera.

Con fines prácticos, la delimitación cartográfica de unidades estructurales se formaliza a través de un mapeo geomorfológico, utilizando como base mapas topográficos y/o fotografías aéreas a diferentes escalas disponibles. Se trabaja de esta manera por cuanto la geomorfología es más visible para fines de delimitación de las unidades, puesto que las diferencias entre tipos de suelos y vegetación, por ejemplo, no se pueden observar con mayor claridad y además porque para estos propósitos se requieren fotografías aéreas y expertos en la materia. En tal sentido, se utilizan como parámetros determinantes las formas del terreno, las pendientes y las elevaciones, para su elaboración en SIG. Según el concepto de tierra, la delimitación de unidades geomorfológicas automáticamente supone también su calificación como unidades ecológicas y de otros tipos a distintos niveles de detalle.

\section{Niveles de detalle}

El Cuadro $\mathrm{N}^{\circ} 1$ muestra un ejemplo de compatibilidad, en términos de detalle, entre tres de los ocho atributos físico-ambientales: suelos, vegetación y geomorfología a diferentes niveles de levantamiento de información para la demarcación de las unidades estructurales (véase también Gráfico $\mathrm{N}^{\circ} 2$ ). Sobra mencionar que una unidad estructural, como lo es un sistema montañoso, es susceptible de subdivisión en unidades más detalladas, es decir en valles, pie de montes, etc., y valles en colinas, terrazas, etc., hasta llegar al nivel de detalle deseado.

Los diferentes niveles de detalle típicamente corresponden a los diferentes niveles de información requeridos por el Gobierno nacional y las autoridades locales, para obtener el panorama general del estado actual de sus territorios. Evidentemente, en una etapa posterior se podrían elaborar, en orden de prioridad, las opciones seleccionadas a nivel de detalle en las diferentes partes de los territorios. Los gobiernos también podrían optar por formular la ordenación de sus territorios en fases -desde el prelevantamiento hasta la factibilidad-. Un ejemplo atañe a los Gobiernos Autónomos Descentralizados Parroquiales Rurales, que no siempre cuentan con presupuestos para estos fines y con personal calificado. En este caso, en una primera fase es pertinente trabajar a nivel de prediagnóstico, pero generando paralelamente algunas respuestas provisionales, como también identificando los vacíos existentes en cuanto a la información requerida. A partir de estos resultados es posible gestionar los fondos necesarios y el personal técnico adecuado para viabilizar los estudios de planificación. 


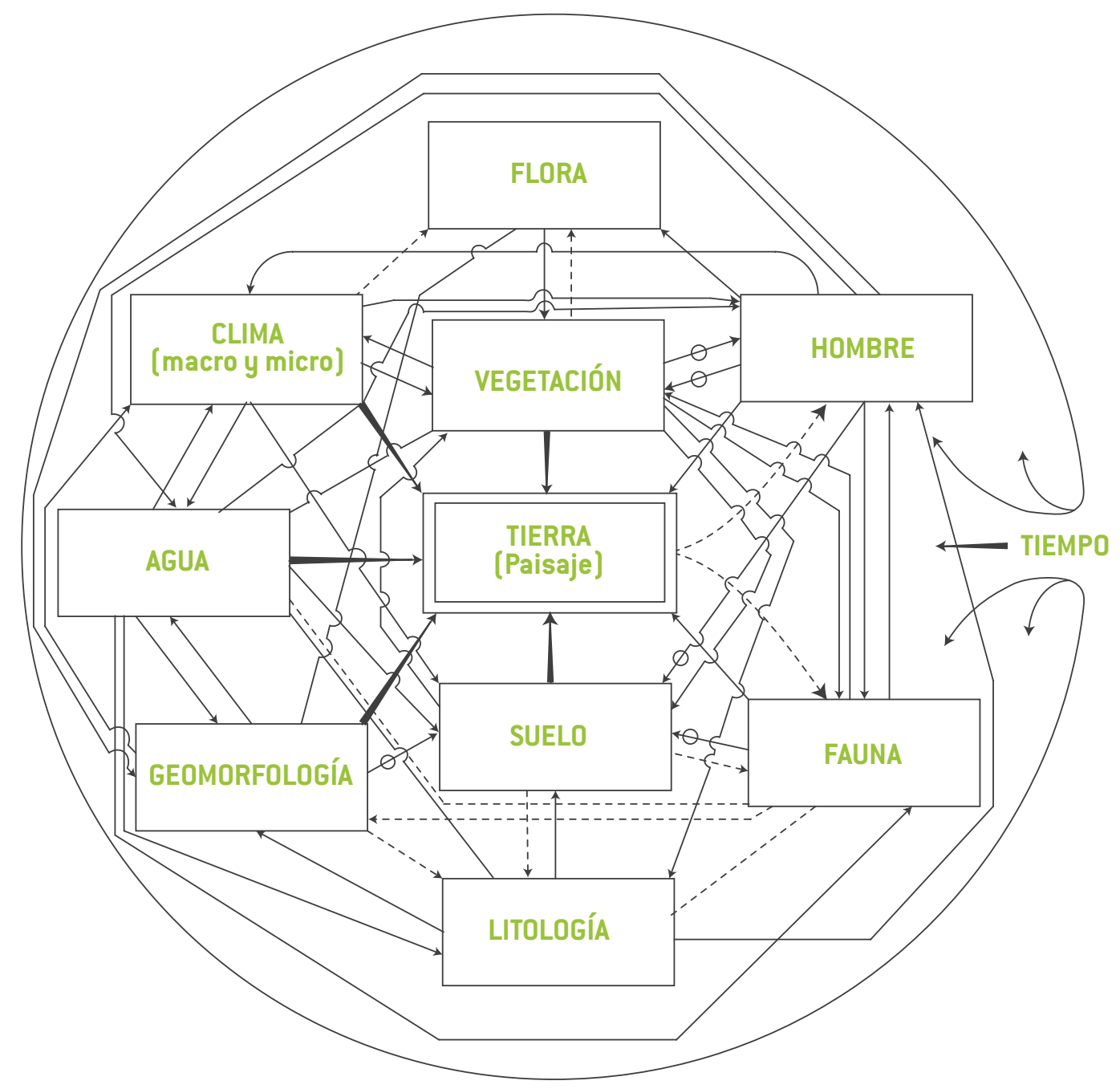

\section{Función físico ambiental de las unidades estructurales}

Las unidades estructurales a través de su capacidad de acogida condicionan las actividades humanas, incluso determinan los límites de su crecimiento territorial. Estas unidades también merecen ser denominadas como zonas agroecológicas que limitan ciertas actividades agropecuarias. Por otro lado, una unidad estructural, o parte de esta unidad, puede representar un acuífero o un ojo de agua que alimenta a un pueblo con agua potable y así pone límites a su crecimiento. De igual manera, el tipo de suelo condiciona la construcción de carreteras y edificaciones, mientras el terreno condiciona las vías de acceso.

Es evidente, entonces, que por medio del concepto de tierra antes aludido, es posible establecer la capacidad de cabida de cada unidad estructural o el conjunto de unidades estructurales de un territorio, como también su biocapacidad, es decir la definición de los límites de su sostenibilidad ambiental en relación a la llamada "huella ecológica". 


\section{Diferencias entre unidades estructurales $y$ unidades ambientales}

Tanto las unidades estructurales como las unidades ambientales se denominan unidades ecológicas. De hecho, es válido que una unidad ambiental sea definida como una "microunidadestructural" o una "unidad estructural parcial" que soporta un determinado uso dado por el hombre y que lo caracteriza; por tanto, una unidad estructural podría comprender una o más unidades ambientales. Otras diferencias entre unidades estructurales y ambientales se refieren a que las primeras se determinan por la disponibilidad y la calidad de recursos como el agua, suelos, microclimas y terrenos para fines específicos y por su biodiversidad, materiales de construcción, energía y paisaje; al mismo tiempo se definen por amenazas como las siguientes: fragilidad ecológica, inestabilidad de laderas, régimen de la lluvia, sequías, huracanes, susceptibilidad a la erosión de los suelos, erupciones volcánicas, etc. Por su lado, las unidades ambientales se caracterizan por las normas de manejo requeridas para su sostenibilidad, es decir por las gestiones necesarias para la prevención de desastres y el manejo de amenazas y riesgos (conservación de suelos, mitigación de inundaciones, estabilidad de laderas, cambio climático, salinización de suelos, contaminación de aguas, desechos sólidos, etcétera).

Vale observar la pertinencia de que las unidades estructurales sean consideradas como una constante en el tiempo a pesar de la existencia de cambios continuos, que sin embargo se producen a un ritmo apenas perceptible. Las unidades ambientales, por otra parte, se deben mirar como variables debido a los cambios de uso de la tierra en un lapso de pocas generaciones, como también por la erosión acelerada de los suelos, los deslaves causados por mal uso, entre otros. En este sentido, es recomendable cuando se planifica el uso de la tierra, que se tome en cuenta la interrelación entre unidades ambientales en su conjunto; por ejemplo, por medio de un enfoque de cuenca con referencia a sus dependencias y complementariedades, en vez del establecimiento de su capacidad de acogida solamente por unidades ambientales individuales.

Fuente: Sebastián Astudillo Cordero 


\section{Función}

\section{socioeconómica de las unidades estructurales}

Mientras las unidades estructurales responden a criterios cartográficos, éstos ya no son aplicables a la delimitación de zonas socioeconómicas. En general, los límites entre estas zonas son difusos y subjetivos. No obstante, en la gran mayoría de los casos se encuentra una estrecha relación entre unidades estructurales -es decir físico-ambientales- y niveles y tipos de actividades socioeconómicas dentro de sus límites. Por ejemplo, no es ninguna sorpresa que la planicie fértil de la costa del Pacífico se caracterice por una economía que se sustente en los cultivos de banano, caña de azúcar, cacao y en la producción de camarones; mientras los páramos se encuentren despoblados por la falta de oportunidades económicas; y que además, la base de la economía de ciudades como Cuenca, históricamente se deba a su posición estratégica en la llamada Ruta Andina. Así que, cada unidad estructural representa una densidad poblacional, uso de la tierra, distintos niveles de ingresos, equipamientos, servicios, densidad vial, etcétera.

Las unidades estructurales a nivel macro por otro nombre y sensu lato también se pueden llamar "Zonas Ecológicas Económicas" -enfoque ZEE-. Dicho enfoque se utiliza ya en varios lugares de Ecuador y otros países y pone énfasis en la definición territorial del ámbito socioeconómico; sin embargo, algunos criterios para la definición adecuada del subsistema físico-ambiental en su mayor parte son obviados. Esto inevitablemente conducirá a errores técnicos en la planificación, particularmente en los aspectos de manejo y gestión ambiental; es decir, dichas interpretaciones, sobre todo subjetivas, generarán incompatibilidades entre los planes de los distintos niveles de ordenación territorial: provincial, cantonal y parroquial rural, debido a los diferentes criterios de definición; lo cual sobre todo impedirá tener una sobrevista para priorizar intervenciones de interés regional o nacional. Por lo tanto, es altamente recomendable utilizar el concepto tierra aquí descrito como punto de partida para la ordenación territorial en todo el país.

\section{Una aplicación: las unidades estructurales y ambientales del cantón Déleg, provincia del Cañar}

Como parte de la formulación -en calidad de trabajo docente (4)-, del Plan de Ordenación Territorial del cantón Déleg, y más concretamente del análisis y diagnóstico de su medio físico, siguiendo los lineamientos metodológicos antes explicitados, se determinaron las correspondientes unidades estructurales en atención a los atributos físico-ambientales: geomorfología, pendientes y elevaciones -en términos de zonas de vida-, obteniéndose 24 unidades (como muestra el Gráfico $\mathrm{N}^{\circ} 3$ ) y en función de ellas se estableció luego la capacidad de acogida para las actividades humanas que el plan estima necesario ordenar a futuro. De hecho, y con fines del análisis y diagnóstico antes aludido, a estas unidades estructurales se adicionó la "capa de uso de suelo", esto es, la información que da cuenta de las actividades humanas que ya soporta el medio físico y su distribución espacial, obteniéndose 88 unidades ambientales que se muestran en el Gráfico $\mathrm{N}^{\circ} 4$ y que en cambio permiten conocer, fundamentalmente, las características naturales de orden funcional del territorio y los modos en que se utiliza y aprovechan sus recursos, incluyendo las degradaciones y amenazas que intervienen sobre el mismo.

Gráfico N³: Cantón Déleg. Provincia del Cañar: Unidades estructurales del medio físico

Fuente: González, Mónica y Ximena Salazar. 2011. "Plan de Ordenación Territorial del cantón Déleg”. Trabajo del Curso-Taller de la Maestría de Ordenación Territorial, Facultad de Arquitectura y Urbanismo de la Universidad de Cuenca. 
Unidades estructurales y su importancia en la ordenación del territorio ecuatoriano

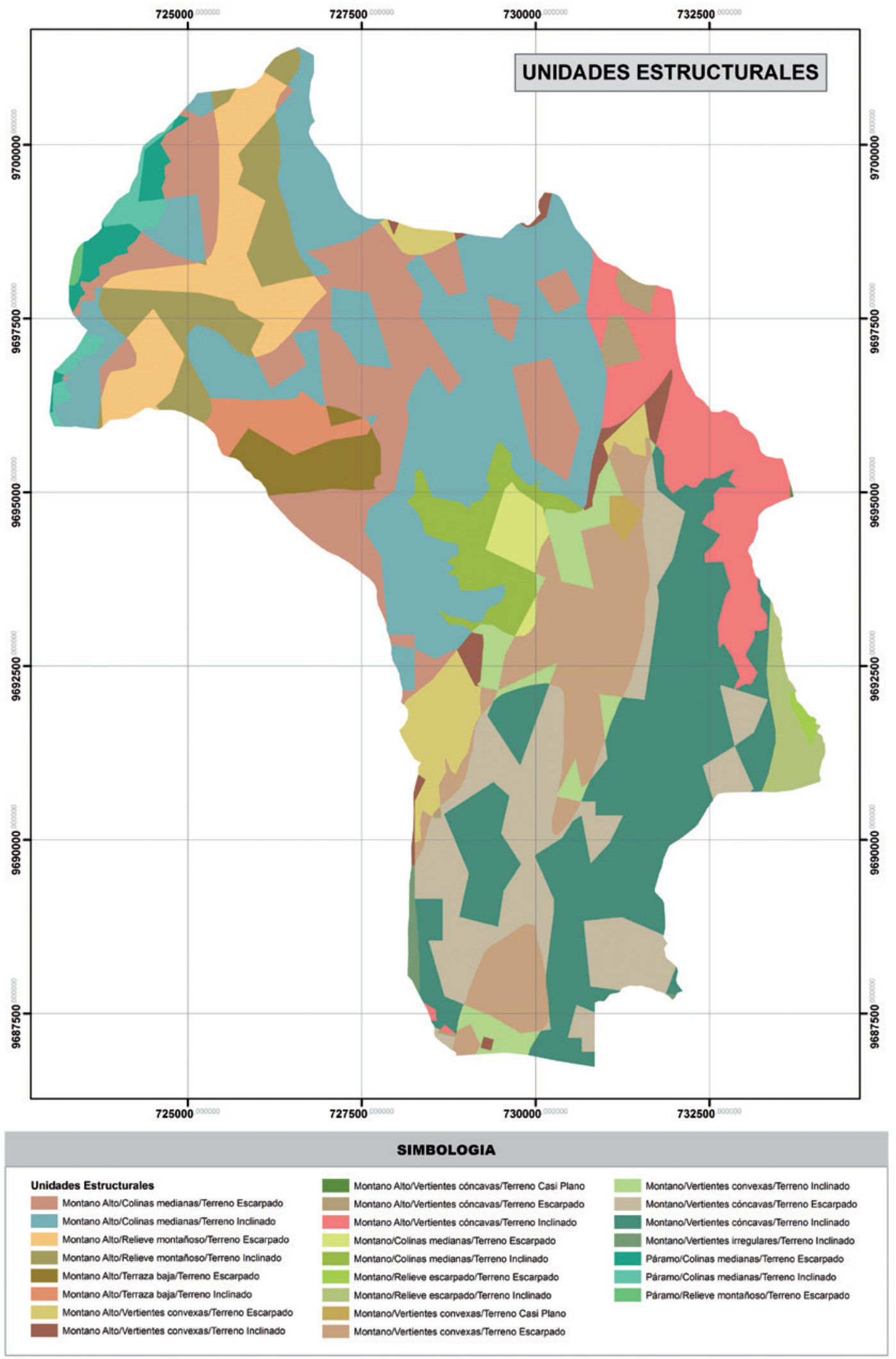



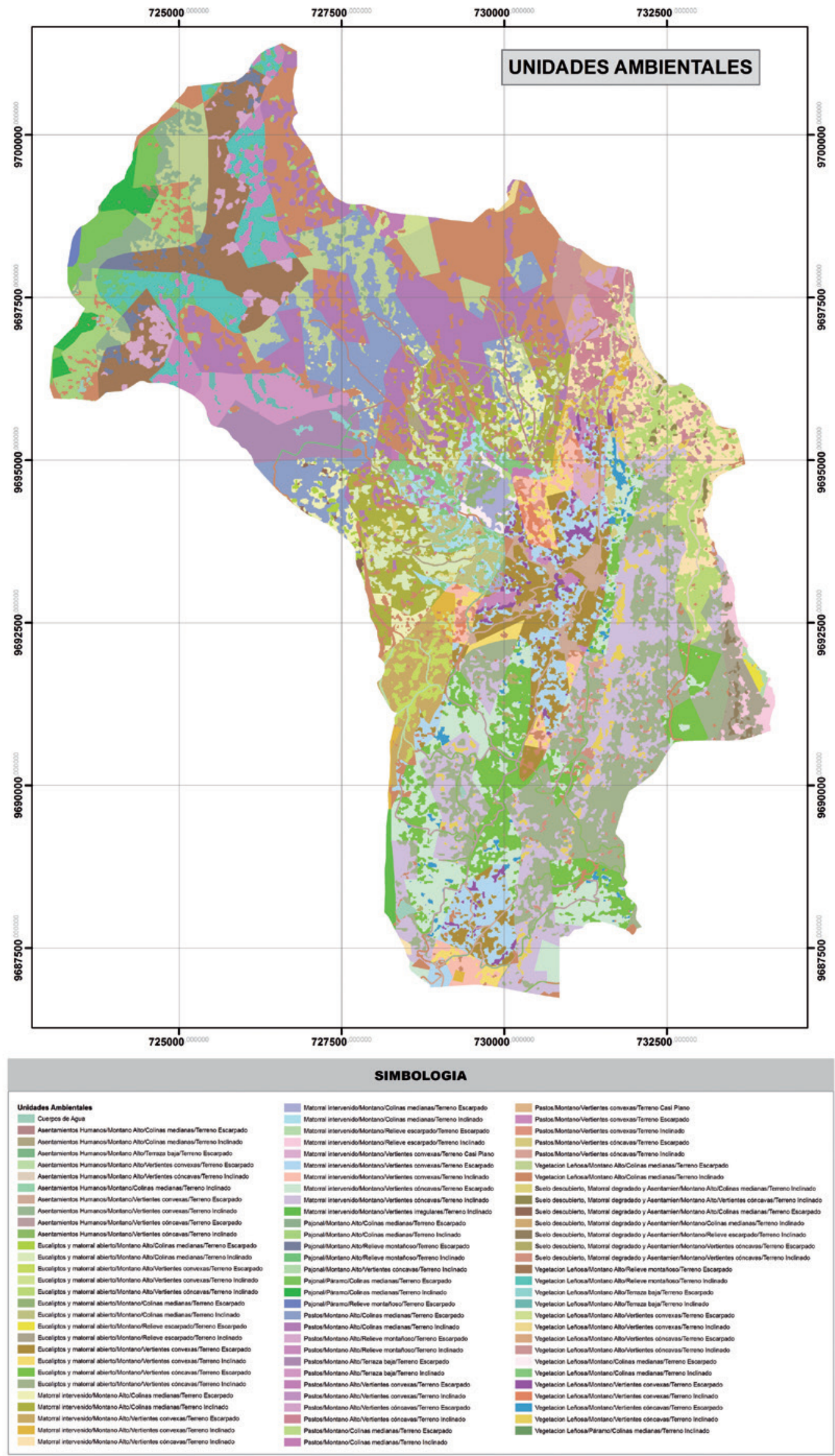
Unidades estructurales y su importancia en la ordenación del territorio ecuatoriano

\section{Bibliografía}

${ }^{1}$ Gómez Orea, Domingo. 2008. “Ordenación Territorial”. Ed. Mundi-prensa, $2^{\circ}$ Edición. Madrid.

2 Ibid, pp. 276 y 277.

${ }^{3}$ ZONNEVELD, I.S. 1972. "Land evaluation and Land (scape) science". ITC Textbook of Photointerpretation Vol.VII. Enschede-Holanda: ITC (International Institute of Aerial Survey and Earth Sciences).

${ }^{4}$ González, Mónica y Ximena Salazar. 2011. “Plan de Ordenación Territorial del cantón Déleg". Trabajo del Curso-Taller de la Maestría de Ordenación Territorial, Facultad de Arquitectura y Urbanismo de la Universidad de Cuenca.

\section{Gráfico $N^{\circ}$ 4: Cantón Déleg. Provincia del Ca- ñar: Unidades Ambientales}

Fuente: González, Mónica y Ximena Salazar. 2011. "Plan de Ordenación Territorial del cantón Déleg”. Trabajo del CursoTaller de la Maestría de Ordenación Territorial, Facultad de Arquitectura y Urbanismo de la Universidad de Cuenca. 